\title{
Measuring the Urban Particulate Matter Island Effect with Rapid Urban Expansion
}

\author{
Yu Cao ${ }^{\circledR}$, Xiaoqian Fang, Jiayi Wang, Guoyu Li, Yu Cao and Yan Li *
}

Department of Land Management, School of Public Affairs, Zhejiang University, Hangzhou 310058, China; caoyu@zju.edu.cn (Y.C.); fangxiaoqian@zju.edu.cn (X.F.); 21822128@zju.edu.cn (J.W.); liguoyu@zju.edu.cn (G.L.); caoyu98@zju.edu.cn (Y.C.)

* Correspondence: liyan522@zju.edu.cn; Tel.: +86-571-56662139

Received: 24 June 2020; Accepted: 27 July 2020; Published: 31 July 2020

\begin{abstract}
Rapid urbanization has posed numerous negative impacts on the environment, including fine particulate matter $\left(\mathrm{PM}_{2.5}\right)$ pollution. However, quantitative investigations of the $\mathrm{PM}_{2.5}$ concentration trends over an urban-rural gradient at the local level are still lacking. The urban particulate matter island (UPI) effect, representing the phenomenon that high particle concentrations in urban areas are gradually attenuated to surrounding areas, was adopted and modified in this paper to study the Hangzhou Bay area from 2000 to 2015. We found the following: (1) every urban area in the Hangzhou Bay area experienced rapid expansion, especially during 2000-2005; (2) more than half of the urban areas suffered UPI problems, and these urban areas had relatively high and stable UPI intensity (UPII) values, although the UPI footprint (UPIFP) values decreased with urban expansion; and (3) urban areas could be divided into three categories: plain areas, hilly areas and the junction of plains and hills, and the probability of the UPI effect varied significantly for different categories. This paper can compensate for the lack of research on the UPI effect at the local level and provide scientific evidence for air pollution control during urban agglomeration planning.
\end{abstract}

Keywords: urban particulate matter island (UPI) effect; air pollution; urban expansion; Hangzhou Bay area

\section{Introduction}

Urbanization, a major anthropogenic modification of Earth, occurs at an unprecedented speed in recorded human history worldwide [1,2]. According to the Department of Economic and Social Affairs of the United Nations Secretariat, more than half of the global population currently lives in urban areas ( $55 \%$, up from $30 \%$ in 1950 ), and by the middle of the century, the proportion is projected to reach $68 \%$. Generally, no other anthropogenic activity alters the environment more radically and persistently than urbanization, and air pollution [3], the urban heat island (UHI) effect [4], global climate change [5], etc. have been proven to be related to rapid urbanization. Among these alarming problems, fine particulate matter with an aerodynamic diameter smaller than $2.5 \mu \mathrm{m}\left(\mathrm{PM}_{2.5}\right)$ has elicited considerable public attention because of its harmful effects on public health. There is convincing evidence that $\mathrm{PM}_{2.5}$ can cause respiratory and cardiovascular problems, lung disease and other health problems [6,7]. Therefore, obtaining a better understanding of $\mathrm{PM}_{2.5}$ pollution control is of great importance. Many studies have investigated meteorological $[8,9]$ and socioeconomic factors $[10,11]$ that may influence the spatiotemporal dynamics of the $\mathrm{PM}_{2.5}$ concentration.

At the same time, urban land has extensively expanded outward across global cities to meet the needs of the soaring number of city dwellers [1]. A worldwide meta-analysis performed by Seto et al. [12] indicated that the urban land area had increased by $58,000 \mathrm{~km}^{2}$ from 1970 to 2000 , with the highest rate of urban expansion observed in India, China, and Africa. Spatiotemporal analysis 
of the urban expansion pattern and dynamics is important not only in urban geography but also in urban planning, environmental, and sustainability studies [13]. Many methods for measuring urban expansion have been proposed. Among these methods, quantitative metrics generated through the size and distribution of new urban land based on land cover maps have been widely applied to assess the physical growth of cities [14,15].

It has been proven that rapid urban expansion exerts many negative impacts on the environment that can transcend far beyond its physical boundary [16]. Among these impacts, the Urban Heat Island (UHI) effect, which is the phenomenon whereby urban areas tend to have a higher temperature than surrounding areas, has been relatively widely researched [2]. In addition to heat energy, cities are also considered a major container of air pollutants. A previous study found that $\mathrm{PM}_{2.5}$ pollution presented similar characteristics to the temperature; thus, a new concept-the urban particulate matter island (UPI) effect-was introduced. Its definition is the phenomenon whereby a higher particle concentration is observed in urban areas that is gradually attenuated to surrounding areas. In addition, two metrics-UPII (UPI intensity) and UPIFP (UPI footprint)—-were designed to quantitatively delineate the UPI phenomenon of 338 prefectures throughout China [17].

$\mathrm{PM}_{2.5}$ data can be divided into two categories-ground monitoring site data and remote sensing data-according to the data source. For the data obtained from ground monitoring sites in China, the $\mathrm{PM}_{2.5}$ concentration was incorporated in the monitoring system by the National Atmospheric Environment Monitoring System in 2012, and national monitoring of the $\mathrm{PM}_{2.5}$ concentration was absent until 2013. Therefore, it is not feasible to use ground observation data for studies on the $\mathrm{PM}_{2.5}$ pollution in China before 2013. In addition, due to the uneven coverage of the ground monitoring sites, limited data are available for studying the spatial and temporal distribution features of $\mathrm{PM}_{2.5}$. Luckily, satellite-based observations can complement the existing network of ground monitoring stations. $\mathrm{PM}_{2.5}$ concentrations, however, cannot be measured directly by satellite imagery. Numerous studies have demonstrated that the aerosol optical depth (AOD) obtained by using satellite instruments, such as Moderate-resolution Imaging Spectroradiometer (MODIS) and Multi-angle Imaging Spectroradiometer (MISR), exhibited a notable correlation with surface $\mathrm{PM}_{2.5}$ concentrations through statistical and mathematical models [18-21]. Owing to the improvements in remote sensing technology, the $\mathrm{PM}_{2.5}$ concentration data estimated by satellite AOD products have been widely used in research [22-24].

However, the rationale of the UPI effect and the interaction between cities have been ignored in previous research. To improve the model of the UPI effect, three areas (urban, surrounding, and background areas) and three $\mathrm{PM}_{2.5}$ processes (emission, dispersion, and deposition) are investigated in this paper. Therefore, all analyses of the UPI effect can be pinpointed to specific areas and processes. The surrounding and background areas constantly changed with the urban area due to urban expansion. Moreover, studies on the UPI effect at the local level are still lacking. To bridge this gap, the Hangzhou Bay area, an urban agglomeration that has experienced rapid urbanization and has suffered severe $\mathrm{PM}_{2.5}$ pollution over the past two decades, was selected as the study area. Considering that the county or district of every prefecture is the most basic economic and social unit in China, the UPI effect of every county or district in the Hangzhou Bay area was studied in this paper. The Hangzhou Bay area is an area with many cultural heritages, such as the West Lake, Liangzhu cultural sites, and numerous museums. $\mathrm{PM}_{2.5}$ pollution can have a certain impact on cultural heritage. Therefore, this paper analyzed the pollution island effect of the Hangzhou Bay area on a relatively macro scale. In this study, a total of 27 urban areas were extracted from the Hangzhou Bay area in 2000, 2005, 2010, and 2015. Then, the occurrence of the UPI effect was determined-the urban areas whose $\mathrm{PM}_{2.5}$ concentration gradually attenuated to the surrounding buffer zones were regarded as areas experiencing the UPI effect. In addition, the spatiotemporal dynamics of UPII and UPIFP were analyzed. Finally, we evaluated the results in terms of the emission, dispersion, and deposition processes. This study aims to answer the following three questions: (1) does the UPI effect exist in the Hangzhou Bay area? (2) If so, what is its magnitude and extent? (3) Is there any regularity in the UPI effect? 
By dividing the study area into urban, surrounding, and background areas, this paper regarded the study area as a whole. By dividing the dynamic processes of pollutants into emission, dispersion, and deposition, this paper analyzed the UPI effect at the process level. By choosing an urban agglomeration as the study area, the UPI effect was studied at the local level. Although the Hangzhou Bay area is just a small part of eastern China, it is a typical urban agglomeration suffering severe $\mathrm{PM}_{2.5}$ pollution, with both plains and hills. Hence, this paper is not just a case study in the Hangzhou Bay area, because the methods can be widely applied to air pollution control during urban agglomeration planning. Compared with existing models, the modified model in this paper can help to analyze the spatial heterogeneity of the UPI effect from the mechanism perspective.

\section{Study Area and Materials}

\subsection{Study Area}

Hangzhou Bay is in the coastal area of southeastern China (Figure 1a), which is both the bay and the entrance to the Qiantang River. In this paper, the Hangzhou Bay area refers to five prefecture-level cities in Zhejiang Province (including Hangzhou, Ningbo, Shaoxing, Jiaxing, and Huzhou, as shown in Figure 1b), which is part of the Yangtze River Delta region. According to the data provided by the Zhejiang Statistics Bureau, the Hangzhou Bay area covers an area of $44,988 \mathrm{~km}^{2}$ in 2018, with a total resident population of 30.796 million. As part of the northern subtropical region, the Hangzhou Bay area is characterized by a mild and humid climate with abundant rainfall. The annual average temperature in the Hangzhou Bay area is between 15 and $20^{\circ} \mathrm{C}$. The landforms in the Hangzhou Bay area are diverse, dominated by hills and coastal plains, and the overall terrain is high in the west and low in the east. The land use of the Hangzhou Bay area in 2015 indicates that construction land was mainly concentrated in the coastal plain (Figure 1c).

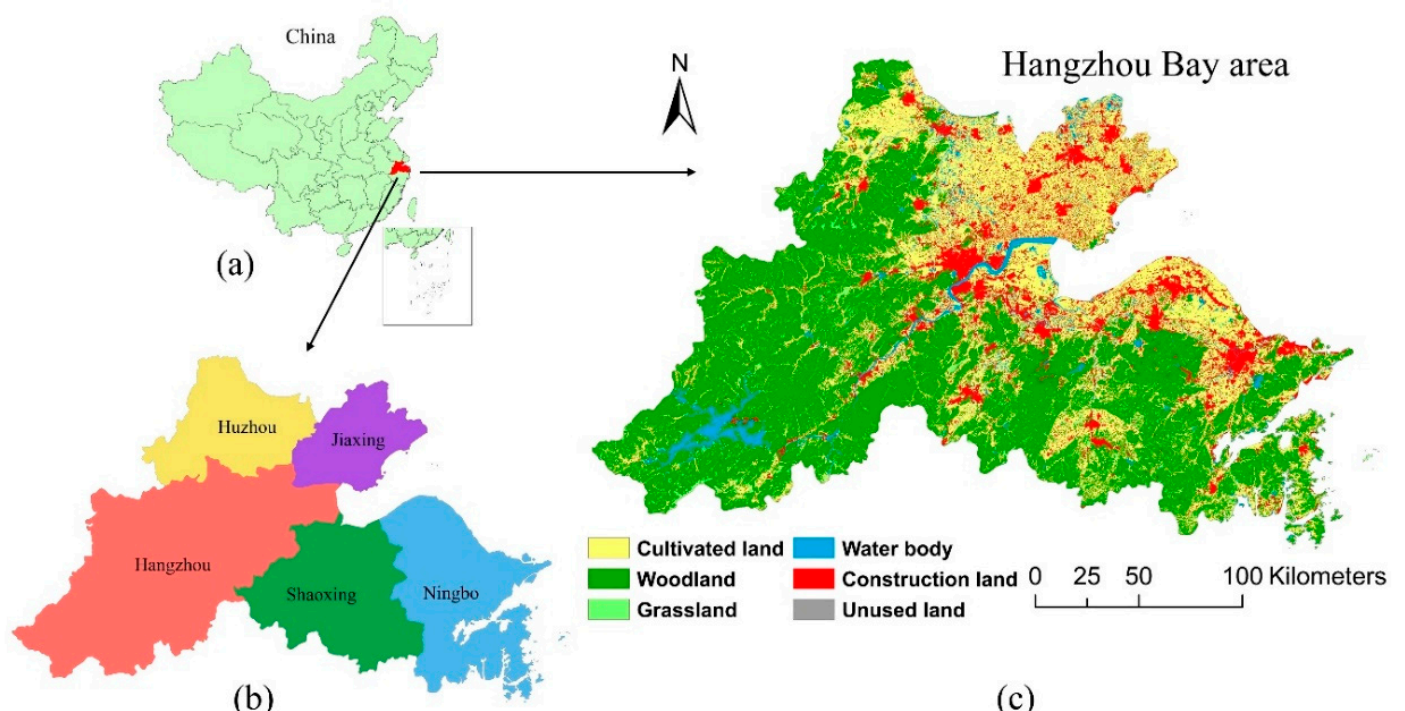

(b)

(c)

Figure 1. (a) Geographical location of the Hangzhou Bay area; (b) five prefecture-level cities in the Hangzhou Bay area; (c) land use of the Hangzhou Bay area in 2015.

As one of the demonstration areas of the reform and opening of China, its geographical conditions and policies make the Hangzhou Bay area an important growth pole for economic and social development not only in the Yangtze River Delta region but also in China as a whole [25]. However, in addition to rapid economic growth and a high urbanization rate, the Hangzhou Bay area has also experienced severe $\mathrm{PM}_{2.5}$ pollution in recent years [11]. In this study, the Hangzhou Bay area was selected as the study area to examine the spatiotemporal dynamics of the UPI effect within the context of rapid urban expansion. 


\subsection{Data Source}

Several datasets, including $\mathrm{PM}_{2.5}$ data, land use data, administrative boundaries, historical Google Earth (Google, Mountain View, CA, USA) images, and digital elevation model (DEM, NASA, Washington, DC, USA) data, are listed in Table 1. The annual mean $\mathrm{PM}_{2.5}$ data were retrieved from the Atmospheric Composition Analysis Group at Dalhousie University (http://fizz.phys.dal.ca/ \{\}atmos/ martin/). The dataset was obtained by combining the aerosol optical depth (AOD, NASA, Washington, DC, USA) retrievals from NASA Moderate-resolution Imaging Spectroradiometer (MODIS, NASA, Washington, DC, USA), Multi-angle Imaging Spectroradiometer (MISR, NASA, Washington, DC, USA), and Sea-viewing Wide Field-of-view Sensor (SeaWiFS, NASA, Washington, DC, USA) instruments with the GEOS-Chem (NASA, Washington, DC, USA) chemical transport model and subsequently calibrated against regional ground-based observations of both the total and compositional mass via geographically weighted regression (GWR, ESRI, Redlands, CA, USA). The ground-based $\mathrm{PM}_{2.5}$ measurements of mainland China were obtained from http://beijingair.sinaapp.com/, which were captured by individuals from instantaneous data records on the website of the Chinese Environmental Protection Agency [26,27].

Table 1. Description of the data used in this study.

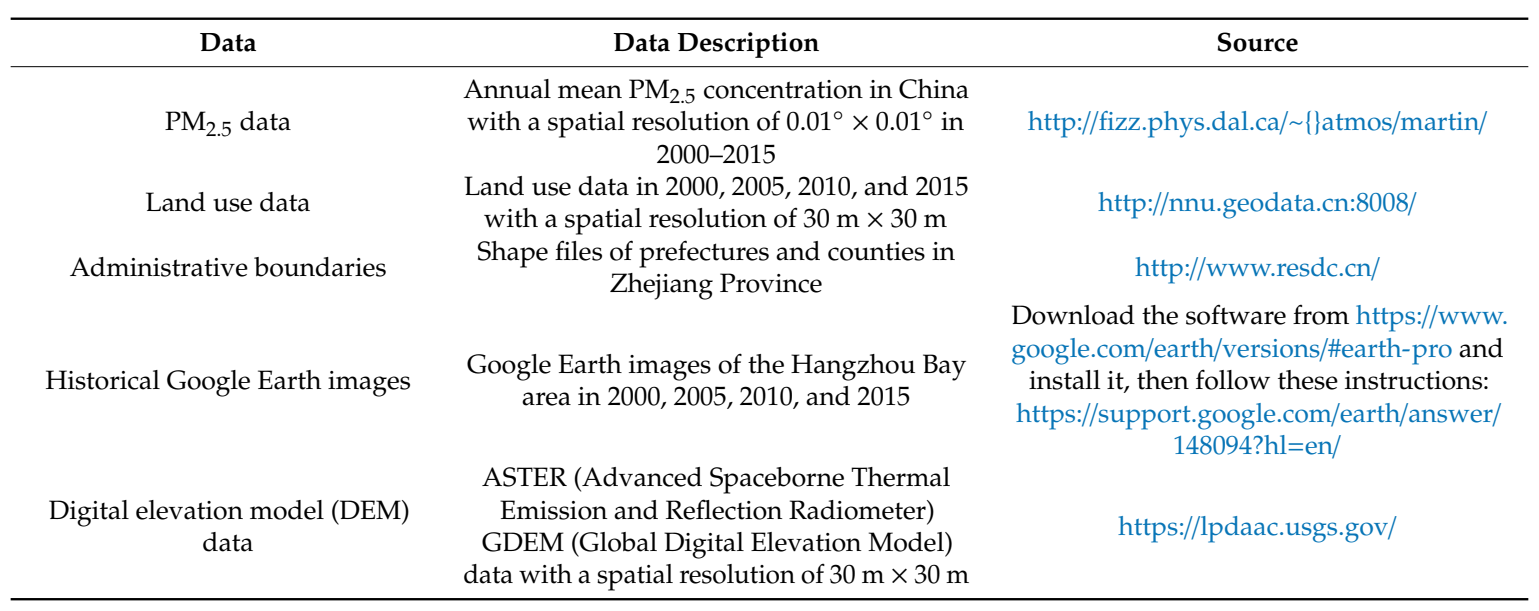

The dataset has been documented with a suitable accuracy on a global scale (the cross-validated $\mathrm{R}^{2}$ value is 0.81 ) [28] and has been widely used [17,29]. Therefore, a subset covering the Hangzhou Bay area in 2000, 2005, 2010, and 2015 was adopted in this study. The data downloaded from the website have the World Geodetic System — the 1984 Coordinate System-with a spatial resolution of $0.01^{\circ}$. The projection transformation, extraction, and zonal statistics tools in geographical information systems (ESRI, Redlands, CA, USA) were implemented to successively process the annual mean $\mathrm{PM}_{2.5}$ concentration data.

Land use data and historical Google Earth images were used to extract the urban area, and historical Google Earth images were supplements to land use data. Unlike the other datasets, the source of historical Google Earth images is the Google Earth Pro software (Google, Mountain View, CA, USA). Moreover, administrative boundaries data and digital elevation model (DEM) data were used in this study.

\section{Methods}

\subsection{Rationale}

The rationale of this paper can be summarized as the emission, dispersion, and deposition of $\mathrm{PM}_{2.5}$. Principal component analysis performed on the aerosol data has revealed that vehicular emissions, coal/biomass combustion, industry sources, soil dust, and secondary formation are the main 
potential sources for the ionic components of $\mathrm{PM}_{2.5}$ in China [30]. The economic growth, urbanization, and industrialization of China have turned urban areas into the main sources and victims of air pollution [31].

By emitting the $\mathrm{PM}_{2.5}$ pollutant into the atmosphere, the process of dispersion results in transboundary air pollution. Dispersion is always accompanied by deposition, which is a process that removes pollutants from the atmosphere and transfers them to the ground surface. After emission, some of the particulates settle due to gravitation, some are eluted from the atmosphere by precipitation, and others remain in the atmosphere for a long time. It has been assumed that meteorological conditions, particle properties, and underlying surface characteristics can affect the dispersion and deposition of $\mathrm{PM}_{2.5}$ [32].

The UPI effect, referring to the phenomenon whereby the high $\mathrm{PM}_{2.5}$ concentrations in urban areas are gradually attenuated to surrounding areas, is essentially a comprehensive manifestation of the emission, dispersion and deposition of $\mathrm{PM}_{2.5}$ (Figure 2b).

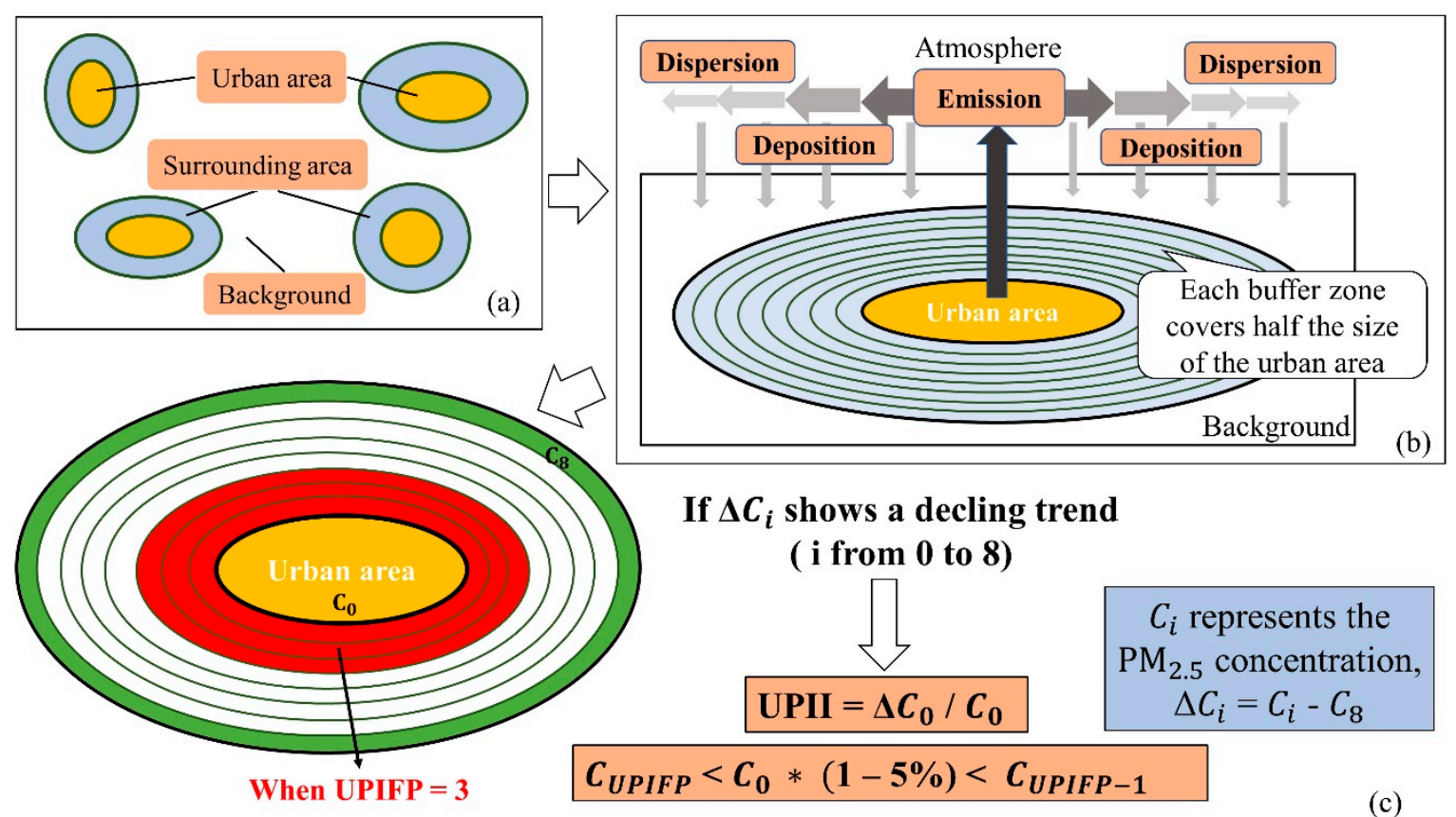

Figure 2. Model of the urban particulate matter island (UPI) effect. (a) Division of the study area; (b) the three processes of particulate emission, dispersion, and deposition; (c) evaluation criterion and two metrics of the UPI effect.

\subsection{Extraction of the Urban and Surrounding Areas}

To determine the occurrence of the UPI effect, the study area was divided into urban, surrounding, and background areas (Figure 2a). Considering that the county/district of every prefecture-level city or municipality is the most basic economic and social unit in China, the urban area in this paper was defined as the urban land of every county or district in these five prefecture-level cities. Based on the land use data and historical Google Earth images, the urban areas in 2000, 2005, 2010, and 2015 were identified and extracted.

Specifically, for each urban area, eight buffer zones surrounding the urban area were generated using geographical information systems software, and each buffer zone covered half the size of the urban area. The purpose of the equal-area buffer zone instead of the equal-distance buffer zone is to ensure that each urban area is comparable. Based on both, the size of each urban area and the distance among the urban areas, eight buffer zones eventually proved to be the best choice. However, some urban areas were geographically very close, leading to overlapping buffer zones. Instead of removing these urban areas directly from this experiment as in a previous study [17], the method of merging was 
applied in this paper, which means that the urban areas with overlapping buffer zones were regarded as one urban area.

To illustrate the above more clearly, the eight districts of Hangzhou (Shangcheng, Xiacheng, Jianggan, Gongshu, Xihu, Binjiang, Xiaoshan, and Yuhang) were selected as an example (Figure 3a). In 2000, Jianggan District and Yuhang District, located northeast of Hangzhou, were relatively far from the other six districts. However, with rapid urban expansion, overlapping buffer zones emerged in 2005. Therefore, all these districts were merged into one urban area. Based on this principle, there are 27 urban areas in the study area, and each urban area has its own corresponding number, such as 1-1 (the first number represents the prefecture-level city) (Figure 3b). In this paper, these numbers were adopted to represent the 27 urban areas in the following sections.

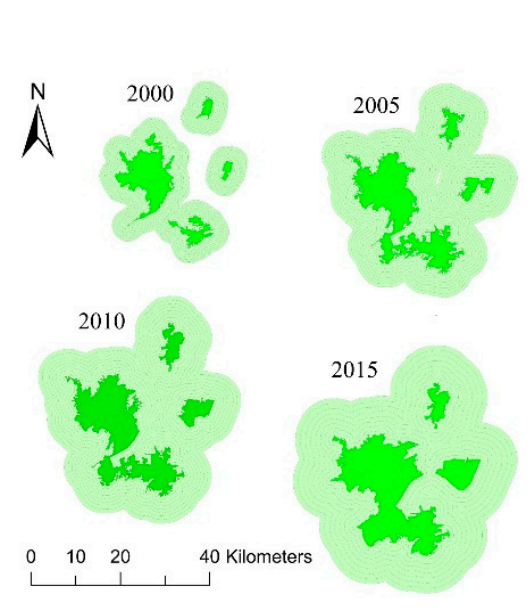

(a)

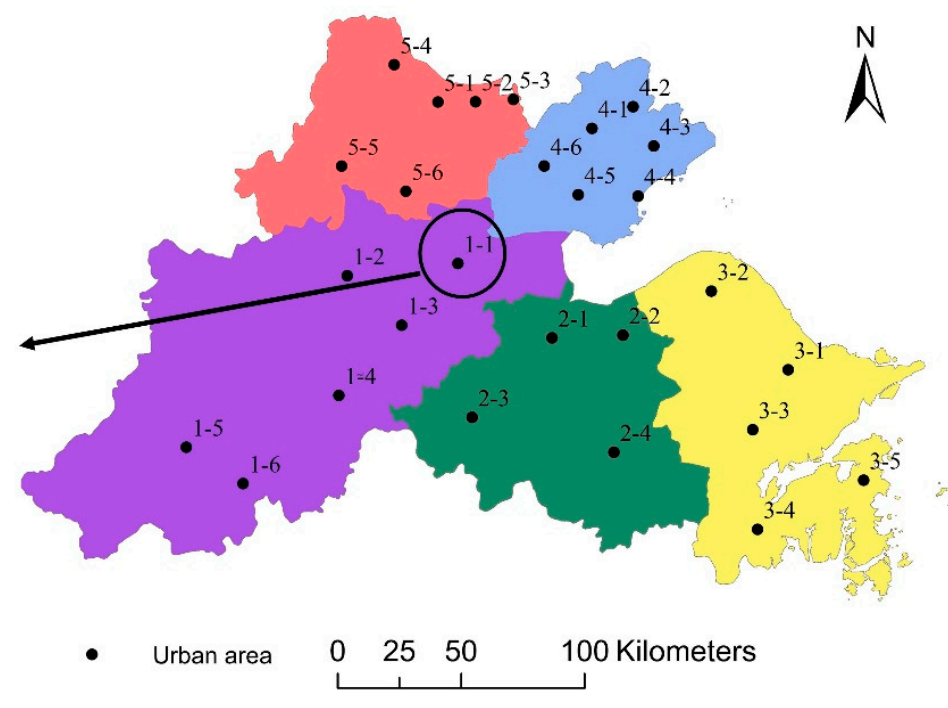

(b)

Figure 3. Urban areas in the Hangzhou Bay area. (a) Urban areas and buffer zones in Hangzhou (urban1-1) in 2000, 2005, 2010, and 2015; (b) location of the 27 urban areas.

\subsection{Model of the UPI Effect}

The average $\mathrm{PM}_{2.5}$ concentration in each urban and buffer was calculated. For each urban area, $\Delta C_{i}$ was defined as the $P_{2.5}$ concentration difference between the $i$-th buffer zone $C_{i}$ and the outermost buffer zone $\mathrm{C}_{8}$ (the 8 th buffer zone). It was calculated with the following Equation (1):

$$
\Delta \mathrm{C}_{\mathrm{i}}=\mathrm{C}_{\mathrm{i}}-\mathrm{C}_{8}, \mathrm{i}=\{0,1,2 \ldots, 8\}
$$

where $C_{0}$ (when $i=0$ ) is the $P_{2.5}$ concentration of the urban area, and $C_{i}$ is the $P_{2.5}$ concentration of the $\mathrm{i}$-th buffer zone. $\Delta \mathrm{C}$ is a series of differential values for the urban area and all its buffer zones. The trends of $\Delta C$ for each urban area in 2000, 2005, 2010, and 2015 were analyzed to examine the attenuation of the $\mathrm{PM}_{2.5}$ concentration. The urban area whose $\mathrm{PM}_{2.5}$ concentration was gradually attenuated from the urban area to the surrounding buffer zones was regarded as an urban area experiencing the UPI effect.

To quantitatively delineate the UPI effect and its spatiotemporal pattern, two metrics have been defined. The first metric is the UPI intensity (UPII), which reflects the magnitude of the UPI effect and can be formulated in Equation (2) as follows:

$$
\mathrm{UPII}=\Delta \mathrm{C}_{0} / \mathrm{C}_{0}
$$


This means that the $\mathrm{PM}_{2.5}$ concentration ratio decayed from the urban area to the outermost buffer zone. In this paper, 0.1 is set as the UPII threshold. Considering $\mathrm{PM}_{2.5}$ standard from the World Health Organization (WHO), urban areas with $\mathrm{C}_{0}>10 \mu \mathrm{g} / \mathrm{m} 3$ and UPII $>0.1$ are regarded as those with a high UPII. It is well known that urban pollution stems from both natural and anthropogenic sources [33]. Therefore, by removing the influence of the natural background, UPII can be viewed as the urban pollution caused by anthropogenic activities [17].

The second metric, the UPI footprint (UPIFP), was defined as the number of buffer zones across which the urban $\mathrm{PM}_{2.5}$ concentration decreases by $5 \%$ (half of the UPII threshold) from the urban area. It can be calculated in Equation (3) as follows:

$$
\mathrm{C}_{\text {UPIFP }}<\mathrm{C}_{0} \times(1-5 \%)<\mathrm{C}_{\text {UPIFP-1 }}
$$

UPIFP quantifies the extent of the continuous effects from the urban area to the surrounding areas. In this paper, UPIFP is an integer ranging from 1 to 8 (Figure 2c). A higher UPIFP value indicates that urban pollution is transported to a further buffer zone. In other words, the higher the value of UPIFP is, the lower the degree of $\mathrm{PM}_{2.5}$ accumulation in the urban area is compared to that in the surrounding area, and the less difficult it is for $\mathrm{PM}_{2.5}$ pollution to spread outward. In this paper, urban areas with UPIFP values higher than 4 are regarded as areas with a large pollution $\mathrm{PM}_{2.5}$ effect range.

The overall concept of this paper is shown in Figure 2. Based on the rationale mentioned above, the method can be defined as according to several steps: (1) extract the urban areas; (2) generate the equal-area buffer zones as the surrounding areas; (3) calculate the average $\mathrm{PM}_{2.5}$ concentration in each urban and surrounding area; (4) analyze the concentration difference from the urban area to the outermost buffer zone; (5) determine whether the UPI phenomenon exists or not; and (6) if so, calculate the UPII and UPIFP of each urban area.

\section{Results}

\subsection{Spatial Patterns of Urban Expansion}

The urban expansion that occurred in the Hangzhou Bay area from 2000 to 2015 is shown in Figure 4a. The total area of the extracted urban areas was $470.41 \mathrm{~km}^{2}$ in $2000,1087.96 \mathrm{~km}^{2}$ in 2005 , $1325.15 \mathrm{~km}^{2}$ in 2010 , and $1584.68 \mathrm{~km}^{2}$ in 2015. The expansion that occurred from 2000 to 2005 occupied $55.42 \%$ of the total urban expansion from 2000 to 2015, making 2000-2005 the period with the most rapid urban growth in the Hangzhou Bay area, followed by 2010-2015 (23.29\%), and the period with the least rapid growth was 2005-2010 (21.29\%). Violin plots of the study area and multiple expanded areas are shown in Figure 5a,b, respectively. Combining the kernel density estimation curve and box plot, the violin plots indicated that the median area increased from 2000 to 2015, while the median expanded area decreased over this period. Although violin plots are less common than box plots, they show similar information. In addition to the median and interquartile range, a violin plot shows the distribution of the data with a rotated smoothing curve.

To visualize the expansion of each urban area more clearly, the specific expansion of each urban area is shown in Figure $4 \mathrm{~b}$ (the scale of each urban area varies). Each urban area expanded from 2000 to 2015, but considering the expansion form and size, each urban area differed. In terms of the expansion form, basically, every urban area expanded outward from the original urban area (referring to the urban area in 2000) in all directions over this period without terrain and hydrological restrictions. In addition, the area of each urban area was analyzed as well. According to the calculation of the area, urban1-1 (which is the urban area whose corresponding number is 1-1, the same as below) possessed the largest area all the time, followed by urban3-1. Four urban areas expanded more than three times from 2000 to 2005 , i.e., urban4- 6 expanded by $348.31 \%$, urban4-2 expanded by $659.02 \%$, urban $5-2$ expanded by $369.92 \%$, and urban5-6 expanded by $449.12 \%$. 


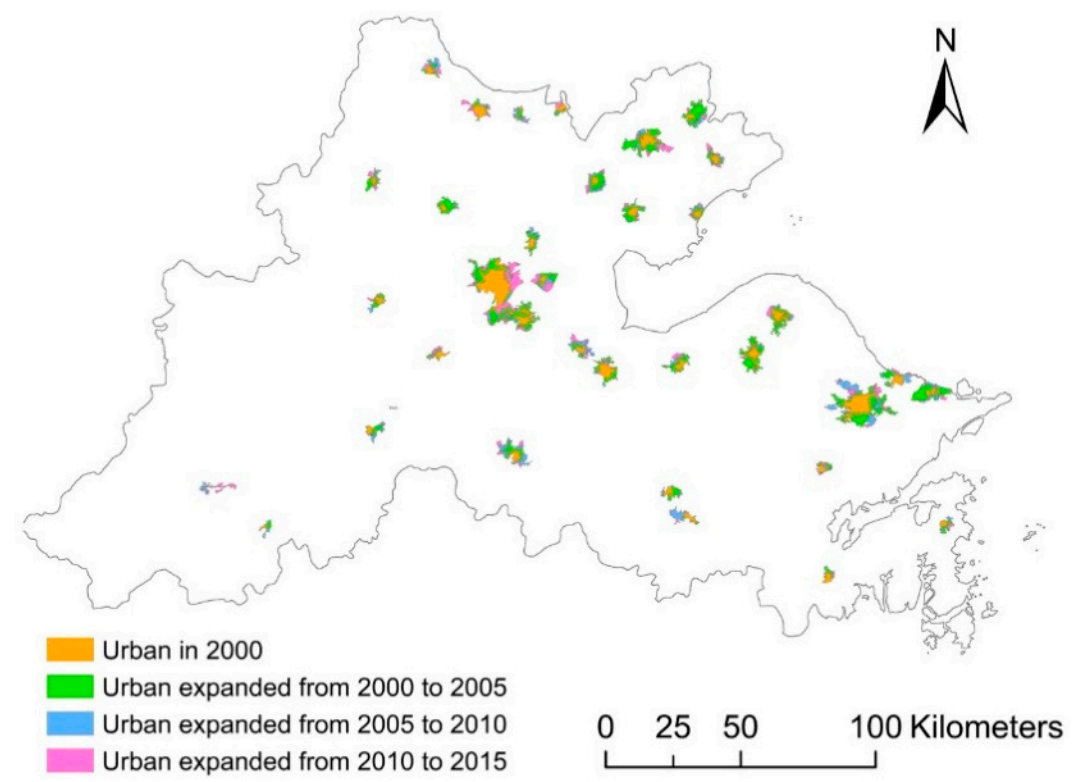

(a)

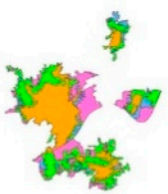

1-1

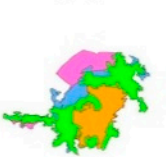

2-2

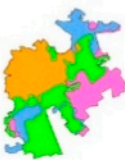

3-5

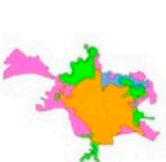

5-1

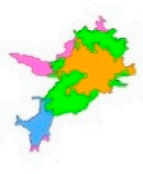

1-2

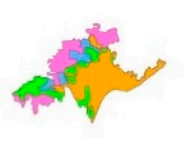

1-3

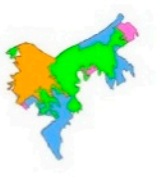

1-4

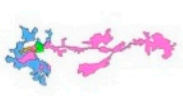

1-5

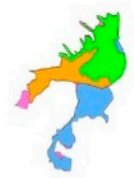

1-6

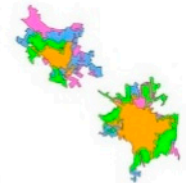

2-1

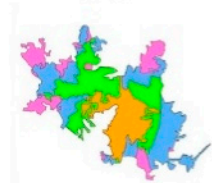

2-3

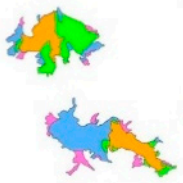

2-4

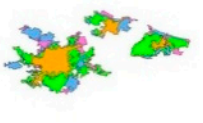

3-1
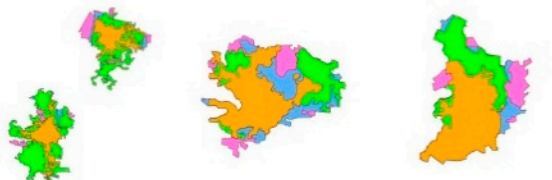

3-2

3-3

3-4
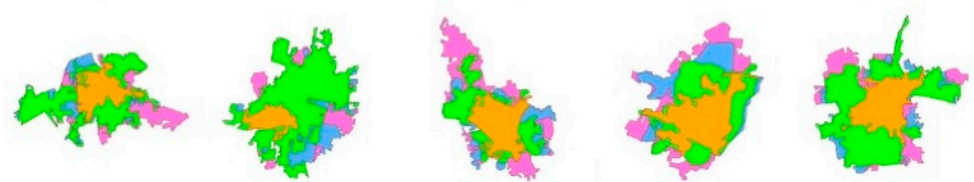

4-5

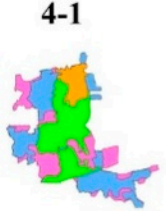

4-2

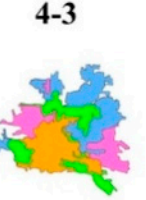

4-4

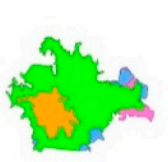

(b)

5-4

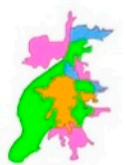

5-5
4-6

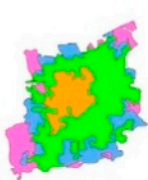

Figure 4. Urban expansion from 2000 to 2015 in the Hangzhou Bay area. (a) All urban areas; (b) each urban area. 

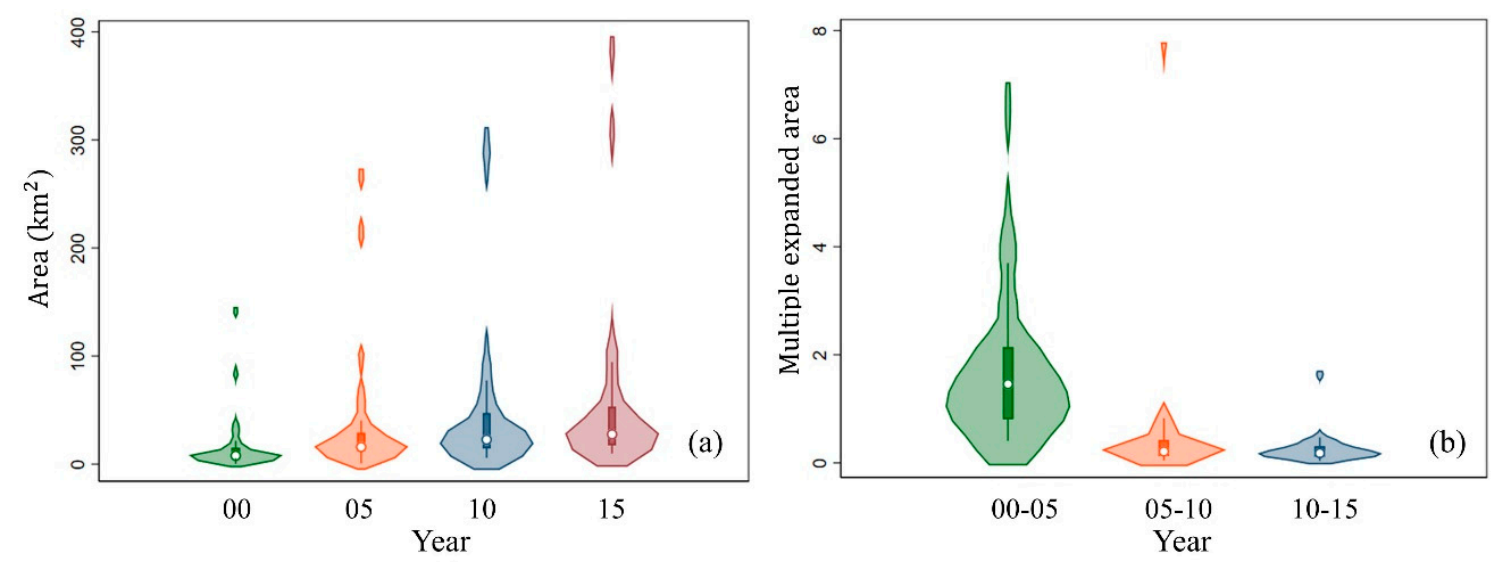

Figure 5. Statistics on the urban expansion of the 27 urban areas in the Hangzhou Bay area. (a) Area in 2000, 2005, 2010, and 2015; (b) multiple expanded areas during 2000-2005, 2005-2010, and 2010-2015.

\section{2. $P M_{2.5}$ Concentration in the Urban Areas and Buffer Zones}

The annual mean $\mathrm{PM}_{2.5}$ concentration within each urban area in 2000, 2005, 2010, and 2015 is shown in Figure 6. From the perspective of the spatial distribution, both the northeast and southwest regions of the Hangzhou Bay area presented relatively low $\mathrm{PM}_{2.5}$ concentrations. In terms of the $\mathrm{PM}_{2.5}$ concentration of each urban area, the $\mathrm{PM}_{2.5}$ concentration in urban1-4, urban1-5, urban1-6, urban3-4, urban3-5, and urban5-5 remained low from 2000 to 2015, while that in urban1-1 remained high throughout in this period. In addition, the cluster formed by urban4-1, urban4-2, urban4-3, urban4-4, urban4-5, and urban4-6 experienced a notable increase in $\mathrm{PM}_{2.5}$ concentration from 2000 to 2015. These six urban areas are all located in Jiaxing and are geographically close to one another.

By evaluating the $\mathrm{PM}_{2.5}$ concentrations in these four years, the most significant point is that the $\mathrm{PM}_{2.5}$ concentrations in 2000 were considerably lower than that in the other three years. The minimum concentration in 2000 was $25.81 \mu \mathrm{g} / \mathrm{m}^{3}$, while it was $36.12 \mu \mathrm{g} / \mathrm{m}^{3}$ in $2005,36.37 \mu \mathrm{g} / \mathrm{m}^{3}$ in 2010 and 34.16 $\mu \mathrm{g} / \mathrm{m}^{3}$ in 2015. At the same time, the maximum concentration in 2000 was $54.03 \mu \mathrm{g} / \mathrm{m}^{3}$, while it was $65.75 \mu \mathrm{g} / \mathrm{m}^{3}$ in $2005,59.31 \mu \mathrm{g} / \mathrm{m}^{3}$ in 2010 , and $58.57 \mu \mathrm{g} / \mathrm{m}^{3}$ in 2015 . In summary, both the minimum and maximum concentrations were the lowest in 2000.

Regarding each urban area, $\triangle \mathrm{C}$ (the $\mathrm{PM}_{2.5}$ concentration difference between the urban area/buffer zone and the outermost buffer zone) of the 27 urban areas in 2000, 2005, 2010, and 2015 are shown in Figure $7 \mathrm{a}$. In 15 of the 27 urban areas, $\Delta \mathrm{C}$ clearly decayed from the urban area to the buffer zones, including urban1-1, urban1-2, urban1-3, urban2-1, urban2-2, urban2-3, urban2-4, urban3-1, urban3-2, urban3-3, urban3-4, urban3-5, urban5-1, urban5-4, and urban5-6, which were regarded as urban areas with UPI effects in this paper. The distribution of the urban areas with the UPI effect is shown in Figure $7 \mathrm{~b}$. Except for urban2-2, for each of the remaining 14 urban areas, the shapes of the four curves are roughly similar.

When $\Delta C$ of all 27 urban areas are combined (labeled "all" in Figure 7a), the four decay curves for 2000, 2005, 2010, and 2015 can be obtained. Except for 2000, the curves for the other three years are very similar in both shape and value, and their $\Delta \mathrm{C}_{0}$ values are all higher than 4 . The slope of the attenuation decreases with the distance in 2005, 2010, and 2015. Conversely, $\Delta C_{0}$ in 2000 is 3.55, which is slightly lower than that in the other three years, and the slope of the attenuation remains essentially the same. 

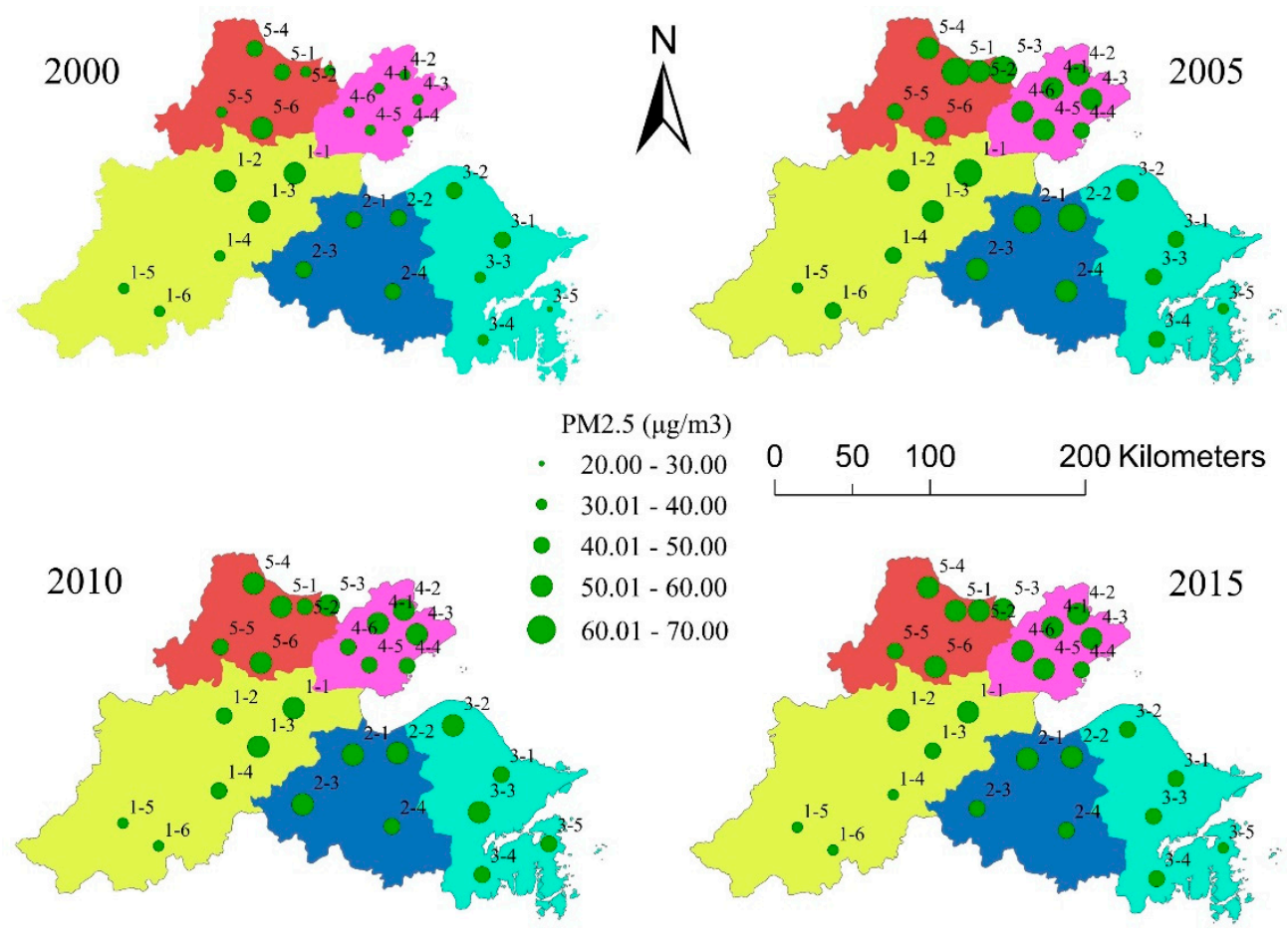

Figure 6. The annual mean fine particulate matter $\left(\mathrm{PM}_{2.5}\right)$ concentration in each urban area.

\subsection{Spatiotemporal Dynamics of UPII and UPIFP}

The spatiotemporal dynamics of the UPII and UPIFP for the urban areas with UPI effects in the Hangzhou Bay area are shown in Figure 8. For comparison purposes, the UPII in the four years are all classified with $0.1,0.15$, and 0.2. Overall, the UPII in each urban area remained relatively stable over time (Figure 9). From the perspective of the spatial distribution, the urban areas with a higher UPII were distributed in the central and southeast regions of the Hangzhou Bay area. The urban area with the highest UPII was urban3-4, whose UPII exceeded 0.2 every year. In addition, the UPII of urban1-1, urban1-2, urban1-3, urban2-1, urban2-3, urban2-4, urban3-1, urban3-2, urban3-3, urban3-5, and urban5-6 were higher than 0.1 in these four years.

In contrast to the UPII, not all urban areas have a UPIFP. Among the 15 urban areas, 14 urban areas have a UPIFP all four years, while urban2-2 only has a UPIFP in 2005, 2010, and 2015. The UPIFP in 2000 includes 1, 2, 3, and 4. In 2005 and 2010, 1, 2, and 3 still occurred, while in 2015, only 1 and 2 remained. The most notable UPIFP phenomenon is that from 2000 to 2015, the UPIFP generally showed a downward trend, especially from 2000 to 2005, which implied that compared to the surrounding area, the $\mathrm{PM}_{2.5}$ accumulation in the urban area became more severe in this period. 

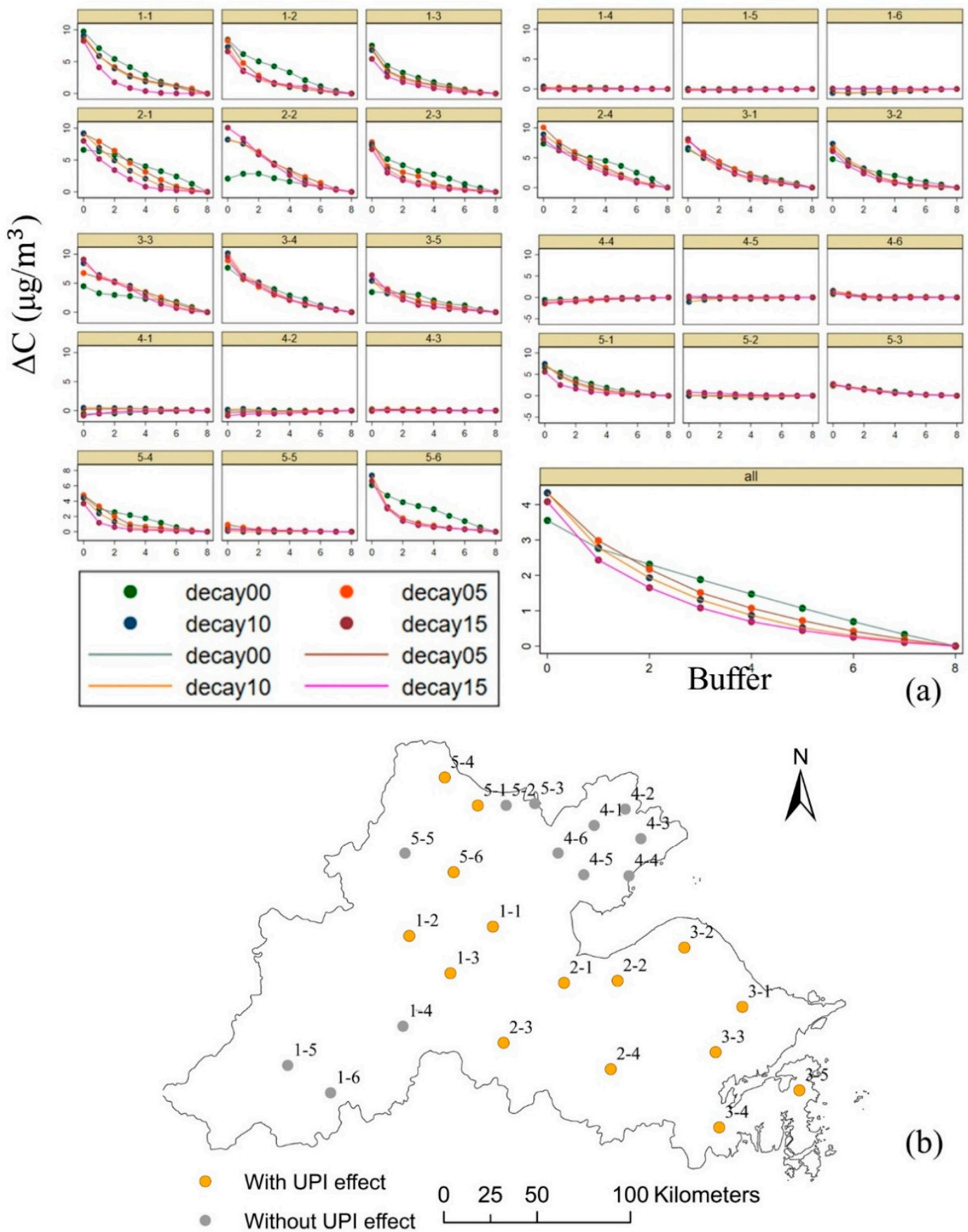

(b)

Figure 7. The occurrence of the UPI effect. (a) Trends of concentration difference $(\Delta C)$ from the urban area to the buffer zones for the 27 urban areas in the Hangzhou Bay area in 2000, 2005, 2010, and 2015; (b) spatial distribution of the urban areas with the UPI effect. 

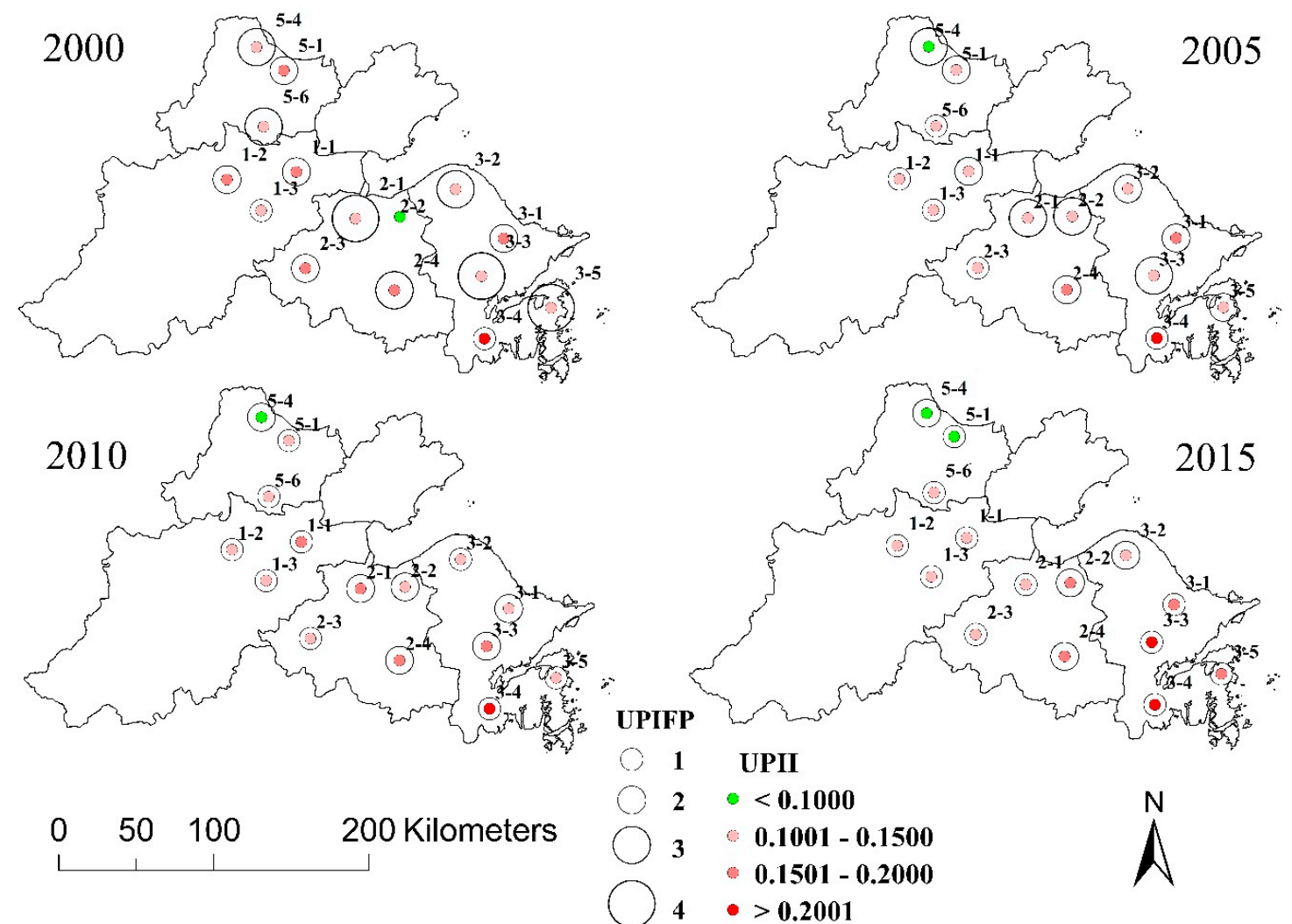

Figure 8. Spatial distribution of the UPI intensity (UPII) and UPI footprint (UPIFP) for the urban areas with UPI effects in 2000, 2005, 2010, and 2015.

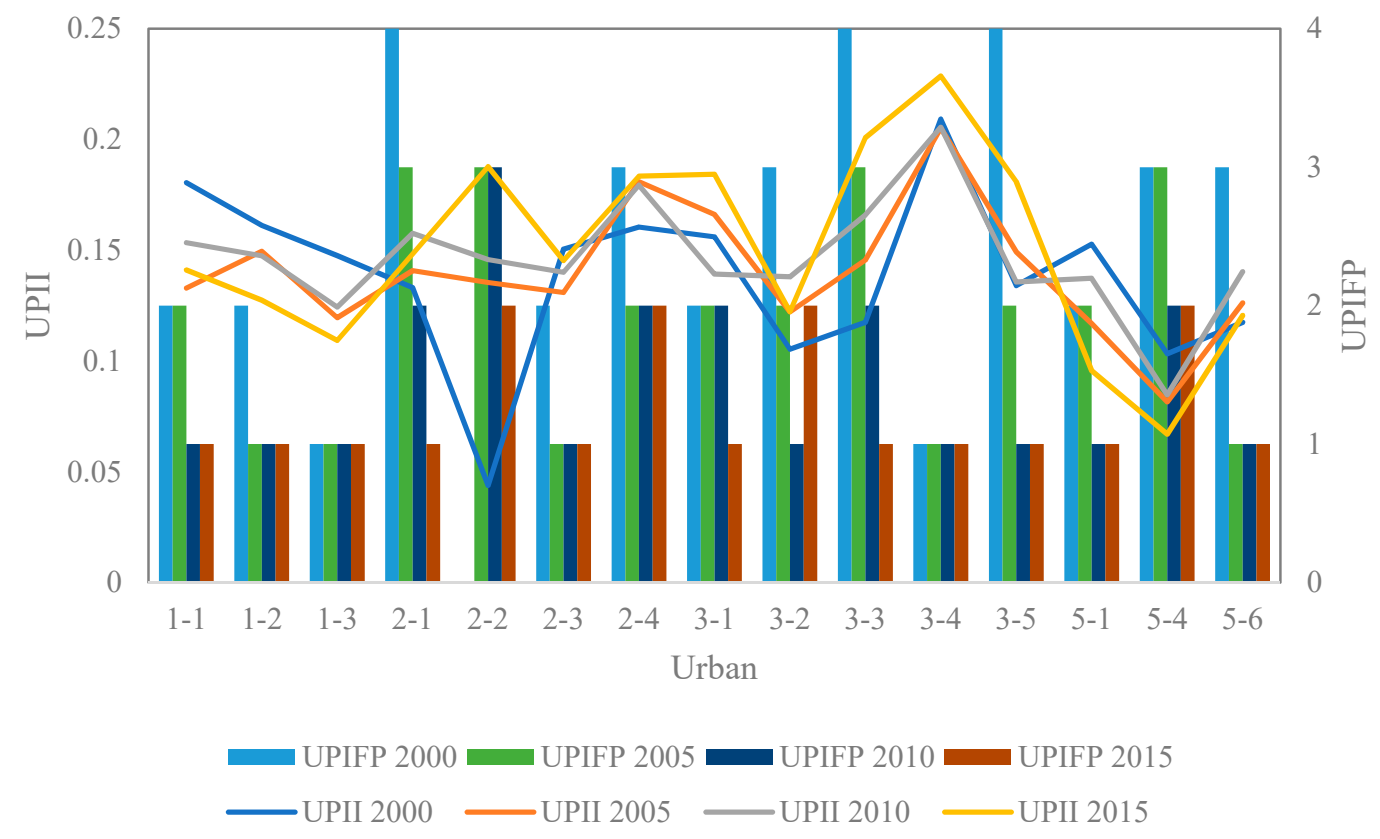

Figure 9. UPI intensity (UPII) and UPI footprint (UPIFP) in 2000, 2005, 2010, and 2015.

\section{Discussion}

\subsection{Emissions}

In this paper, urban areas are considered the source and container of $\mathrm{PM}_{2.5}$ pollution, and the $\mathrm{PM}_{2.5}$ concentration in the urban areas is directly related to local emissions. Because it is the result of 
economic growth, urban expansion can reflect the development of cities to a certain extent. Pearson correlation analysis between the area and $\mathrm{PM}_{2.5}$ concentration of the urban areas indicates that there exists a positive correlation between the area and concentration, especially when the area is smaller than $100 \mathrm{~km}^{2}$ (Figure 10). This is consistent with previous observations [29]. However, the environmental Kuznets curve (EKC) hypothesis claims that there exists an inverted U-shaped connection between environmental degradation and economic growth [34], which means that the Hangzhou Bay area is still at the initial stage of economic growth. Therefore, although $\mathrm{PM}_{2.5}$ pollution can be fundamentally reduced by controlling emissions in the short term, it comes at a significant cost and requires efforts from both the local government and polluting companies [35]. In addition, studies have shown that urban form can influence air pollution in China, and moderately scattered and polycentric urban development is encouraged [36].

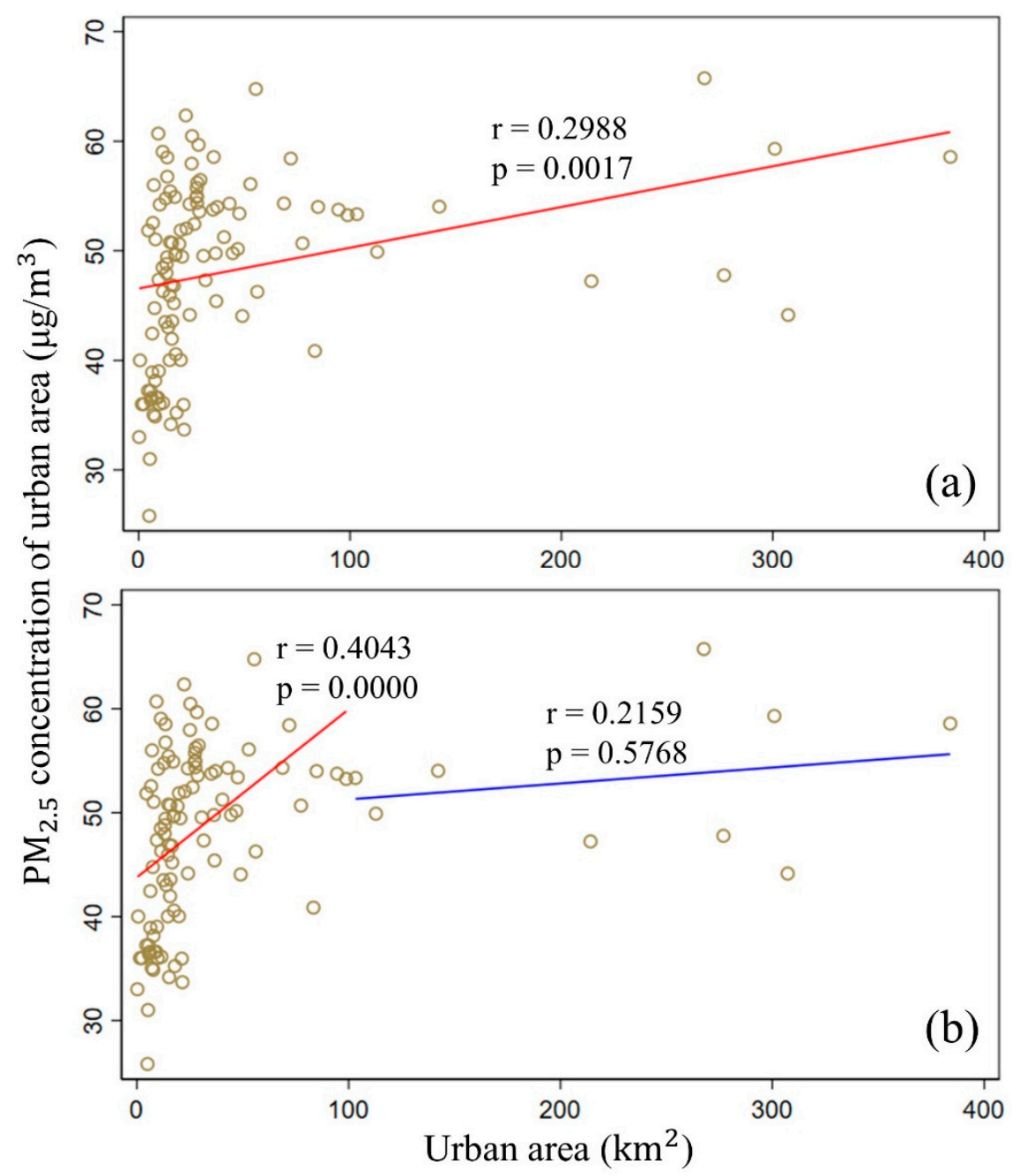

Figure 10. Relationship between the area and $\mathrm{PM}_{2.5}$ concentration in the urban areas. (a) All urban areas; (b) urban areas divided at an area of $100 \mathrm{~km}^{2}$.

\subsection{Dispersion and Deposition}

With increasing economic development and urbanization, the underlying surface of dispersion and deposition has changed. On the one hand, due to the rapid urban expansion, parts of the former surrounding area have become urban areas, and parts of the background area have been transformed into surrounding areas. On the other hand, the land use of the surrounding and background areas has also changed in this period, such as the transition from farmland to rural settlements. To further 
examine the mechanism behind the UPI effect, these 27 urban areas were divided into three categories (Figure 11).

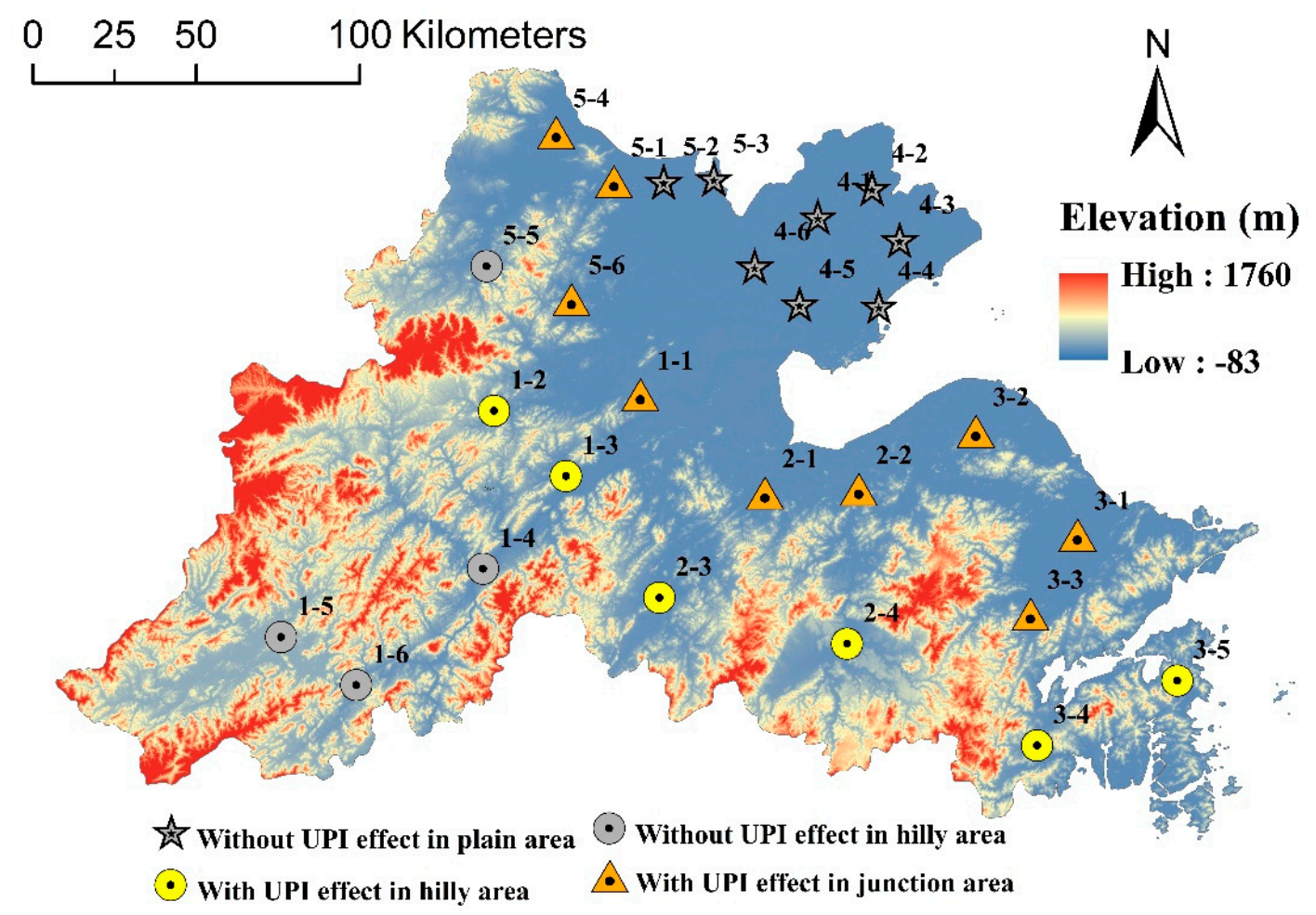

Figure 11. Division of the urban areas by the terrain.

Plain area: the urban areas were distributed in clusters with a high integrity. Due to the flat terrain, cities were generally densely distributed, and urban expansion resulted in a major reduction in the distance between neighboring urban areas, which might enhance the interaction of pollution. In addition, the surrounding and background areas were usually dominated by cultivated land and rural settlements. Therefore, the $\mathrm{PM}_{2.5}$ concentration in the plain area was relatively even, and the probability of the UPI effect was relatively low in this area.

Hilly area: the urban areas were scattered with notable independence. Because of the woodlands in the surrounding areas, the pollutants emitted from the urban areas could be effectively settled. In addition, the large forests in the background areas diminished the effect of urban expansion on the distance between the urban areas. However, due to the terrain, urban expansion was limited, which might make urban pollution accumulation less distinct. Therefore, the probability of the UPI effect was relatively random in this area, and the decrease in UPIFP might be due to the shorter distance between the urban boundaries and woodlands.

Junction of plains and hills: the urban areas were zonally distributed, with both integrity and independence. Compared with the urban areas surrounded by hills, the expansion was relatively less restricted by the terrain but more susceptible to the influence of neighboring urban areas. Compared with the plain area, the surrounding woodlands could effectively settle pollutants. In this paper, the urban areas located in the junction area exhibited a high probability of the UPI effect, and the decrease in UPIFP was also due to the shorter distance between the urban boundaries and woodlands.

\subsection{Main Findings}

In this paper, we proposed a framework to characterize the UPI effect of the Hangzhou Bay area during 2000-2015. The urban areas experienced a rapid expansion during this period. However, the existence of the UPI effect in each urban area differed slightly. More than half of the urban areas 
exhibited the UPI effect, and the existence of the UPI effect was not related to the $\mathrm{PM}_{2.5}$ concentration, but instead to the terrain, and urban areas could be classified into three categories: plain areas, hilly areas, and the junction of plains and hills. Terrain not only affects the dispersion and deposition of pollutants, but also affects the expansion of urban areas, which is the source of emissions. For those urban areas with the UPI effect, the UPII value in each urban area remained relatively stable over time. The UPIFP value generally showed a downward trend, implying that compared to the surrounding areas, $\mathrm{PM}_{2.5}$ accumulation in the urban areas became more severe in this period. Compared with existing literature, at the national level, the concentration of monitoring sites around and within the cities were highly correlated [37]. Remote sensing data have also been used to measure the difference in the $\mathrm{PM}_{2.5}$ concentration between urban and non-urban areas, but without the concentration trends [38]. Some studies focused on only a single megacity: there was no significant difference in concentration between the urban and suburban areas of Shanghai [39]; however, the difference was obvious in Beijing [40]. In this paper, the study area is an urban agglomeration, which between a nation and a single city. Previous studies have generated equidistant buffer zones around cities but did not measure the specific concentration trends of $\mathrm{PM}_{2.5}$ [11]. The impact of land use on the spatial distribution of concentration was previously studied, and forests were found to have the greatest impact on $\mathrm{PM}_{2.5}$ removal [11,41,42], which is consistent with the results in this paper. In general, the results of this paper are consistent with existing literature, and the urban-rural concentration trends on the district/county scale in this paper can provide a theoretical basis for pollution control during urban agglomeration.

\section{Conclusions}

The UPI effect, which refers to the phenomenon whereby the high particle concentration in the urban areas is gradually attenuated to the surrounding areas, was defined and investigated in this paper. The existing literature has revealed that the rationale for the UPI effect is insufficient, and the mutual influence between cities was often ignored. Therefore, this study tried to fill the gap by dividing the study area into urban, surrounding, and background areas and by dividing the dynamic processes of pollutants into emission, dispersion, and deposition. Moreover, to better study the UPI effect at the local level, the urban area in this paper was changed from the urban land of every prefecture to every county or district. As an area with many cultural heritages, the Hangzhou Bay area was selected as the study area. Based on the observations in the 27 urban areas in the Hangzhou Bay area from 2000 to 2015, we examined the urban expansion and the occurrence of the UPI effect for each urban area. The main findings are as follows:

1. Every urban area in the Hangzhou Bay area experienced rapid expansion from 2000 to 2015. For most of the urban areas, 2000-2005 was the period with the most rapid expansion, and several urban areas even attained growth rates above $300 \%$. Although the form and magnitude of urban expansion varied, single-core expansion occurred in most urban areas.

2. By comparison, the $\mathrm{PM}_{2.5}$ concentrations in the urban areas in 2000 were considerably lower than that in 2005, 2010, and 2015. In addition, from the perspective of the spatial distribution, spatial heterogeneity of the $\mathrm{PM}_{2.5}$ concentrations were observed in the Hangzhou Bay area. Overall, the $\mathrm{PM}_{2.5}$ concentration in the urban area had a positive correlation with its area.

3. More than half of the urban areas exhibited the UPI effect in the Hangzhou Bay area during this period. Most urban areas with the UPI effect had a relatively high and stable UPII as well. In contrast to the UPII, the UPIFP values showed a downward trend along with urban expansion.

4. The urban areas could be divided into three categories according to the terrain, i.e., plain areas, hilly areas, and the junction of plains and hills, and the probability of the UPI effect for the different categories varied notably.

Overall, this paper can compensate for the lack of research on the UPI effect at the local level and provide scientific evidence for air pollution control during urban agglomeration planning. 
Author Contributions: Conceptualization, Y.C.1 and Y.L.; methodology, Y.C.1 and X.F.; investigation and software, X.F., J.W., G.L., and Y.C.2; writing-original draft preparation, X.F., Y.C.1; supervision and writing-review and editing, Y.C.1 and Y.L. All authors have read and agreed to the published version of the manuscript.

Funding: This research was funded by the Natural Science Foundation of Zhejiang Province, grant number LY19D010012, and supported by the Fundamental Research Funds for the Central Universities of China.

Conflicts of Interest: The authors declare no conflict of interest.

\section{References}

1. Angel, S.; Parent, J.; Civco, D.L.; Blei, A.; Potere, D. The dimensions of global urban expansion: Estimates and projections for all countries, 2000-2050. Progr. Plan. 2011, 75, 53-107. [CrossRef]

2. Zhou, D.; Zhao, S.; Zhang, L.; Sun, G.; Liu, Y. The footprint of urban heat island effect in China. Sci. Rep. 2015, 5, 1-11. [CrossRef]

3. Rajé, F.; Tight, M.; Pope, F.D. Traffic pollution: A search for solutions for a city like Nairobi. Cities 2018, 82, 100-107. [CrossRef]

4. Li, X.; Zhou, Y.; Asrar, G.R.; Imhoff, M.; Li, X. The surface urban heat island response to urban expansion: A panel analysis for the conterminous United States. Sci. Total Environ. 2017, 60-606, 426-435. [CrossRef] [PubMed]

5. Maheshwari, B.; Pinto, U.; Akbar, S.; Fahey, P. Is urbanisation also the culprit of climate change?-Evidence from Australian cities. Urban Clim. 2020, 31, 100581. [CrossRef]

6. Loftus, C.; Yost, M.; Sampson, P.; Arias, G.; Torres, E.; Vasquez, V.B.; Bhatti, P.; Karr, C. Regional PM2.5 and asthma morbidity in an agricultural community: A panel study. Environ. Res. 2015, 136, 505-512. [CrossRef]

7. Gong, T.; Sun, Z.; Zhang, X.; Zhang, Y.; Wang, S.; Han, L.; Zhao, D.; Ding, D.; Zheng, C. Associations of black carbon and PM2.5 with daily cardiovascular mortality in Beijing, China. Atmos. Environ. 2019, 214, 116876. [CrossRef]

8. Tai, A.P.K.; Mickley, L.J.; Jacob, D.J. Correlations between fine particulate matter (PM2.5) and meteorological variables in the United States: Implications for the sensitivity of PM2.5 to climate change. Atmos. Environ. 2010, 44, 3976-3984. [CrossRef]

9. Liu, W.; Guo, G.; Chen, F.; Chen, Y. Meteorological pattern analysis assisted daily PM2.5 grades prediction using SVM optimized by PSO algorithm. Atmos. Pollut. Res. 2019, 10, 1482-1491. [CrossRef]

10. Wang, S.; Zhou, C.; Wang, Z.; Feng, K.; Hubacek, K. The characteristics and drivers of fine particulate matter (PM2.5) distribution in China. J. Clean Prod. 2017, 142, 1800-1809. [CrossRef]

11. Xu, G.; Ren, X.; Xiong, K.; Li, L.; Bi, X.; Wu, Q. Analysis of the driving factors of PM2.5 concentration in the air: A case study of the Yangtze River Delta, China. Ecol. Indic. 2020, 110, 105889. [CrossRef]

12. Seto, K.C.; Fragkias, M.; Güneralp, B.; Reilly, M.K. A meta-analysis of global urban land expansion. PLoS ONE 2011, 6, e23777. [CrossRef] [PubMed]

13. Sahana, M.; Hong, H.; Sajjad, H. Analyzing urban spatial patterns and trend of urban growth using urban sprawl matrix: A study on Kolkata urban agglomeration, India. Sci. Total Environ. 2018, 628-629, 1557-1566. [CrossRef] [PubMed]

14. Yue, W.; Liu, Y.; Fan, P. Measuring urban sprawl and its drivers in large Chinese cities: The case of Hangzhou. Land Use Pol. 2013, 31, 358-370. [CrossRef]

15. Jiao, L.; Mao, L.; Liu, Y. Multi-order Landscape Expansion Index: Characterizing urban expansion dynamics. Landsc. Urban Plan. 2015, 137, 30-39. [CrossRef]

16. Grimm, N.B.; Faeth, S.H.; Golubiewski, N.E.; Redman, C.L.; Wu, J.; Bai, X.; Briggs, J.M. Global Change and the Ecology of Cities. Science 2008, 319, 756-760. [CrossRef]

17. Huang, X.; Cai, Y.; Li, J. Evidence of the mitigated urban particulate matter island (UPI) effect in China during 2000-2015. Sci. Total Environ. 2019, 660, 1327-1337. [CrossRef]

18. Gupta, P.; Christopher, S.A. Seven year particulate matter air quality assessment from surface and satellite measurements. Atmos. Chem. Phys. 2008, 8, 3311-3324. [CrossRef]

19. Lee, H.J.; Franklin, S.A.; Turkel, J.E.; Goetz, A.R.; Woods, D.W. Facilitated attentional disengagement from hair-related cues among individuals diagnosed with trichotillomania: An investigation based on the exogenous cueing paradigm. J. Obsessive-Compuls. Relat. Disord. 2012, 1, 8-15. [CrossRef] 
20. Chudnovsky, A.; Lyapustin, A.; Wang, Y.; Tang, C.; Schwartz, J.; Koutrakis, P. High resolution aerosol data from MODIS satellite for urban air quality studies. Cent. Eur. J. Geosci. 2014, 6, 17-26. [CrossRef]

21. Chelani, A.B. Estimating PM2.5 concentration from satellite derived aerosol optical depth and meteorological variables using a combination model. Atmos. Pollut. Res. 2019, 10, 847-857. [CrossRef]

22. Yeganeh, B.; Hewson, M.G.; Clifford, S.; Knibbs, L.D.; Morawska, L. A satellite-based model for estimating PM2.5 concentration in a sparsely populated environment using soft computing techniques. Environ. Modell. Softw. 2017, 88, 84-92. [CrossRef]

23. He, Q.; Huang, B. Satellite-based high-resolution PM2.5 estimation over the Beijing-Tianjin-Hebei region of China using an improved geographically and temporally weighted regression model. Environ. Pollut. 2018, 236, 1027-1037. [CrossRef] [PubMed]

24. Gui, K.; Che, H.; Wang, Y.; Wang, H.; Zhang, L.; Zhao, H.; Zheng, Y.; Sun, T.; Zhang, X. Satellite-derived PM2.5 concentration trends over Eastern China from 1998 to 2016: Relationships to emissions and meteorological parameters. Environ. Pollut. 2019, 247, 1125-1133. [CrossRef] [PubMed]

25. Xiao, R.; Lin, M.; Fei, X.; Li, Y.; Zhang, Z.; Meng, Q. Exploring the interactive coercing relationship between urbanization and ecosystem service value in the Shanghai-Hangzhou Bay Metropolitan Region. J. Clean. Prod. 2020, 253, 119803. [CrossRef]

26. Van Donkelaar, A.; Martin, R.V.; Brauer, M.; Boys, B.L. Use of satellite observations for long-term exposure assessment of global concentrations of fine particulate matter. Environ. Health Perspect. 2015, 123, 135-143. [CrossRef]

27. Van Donkelaar, A.; Martin, R.V.; Li, C.; Burnett, R.T. Regional Estimates of Chemical Composition of Fine Particulate Matter Using a Combined Geoscience-Statistical Method with Information from Satellites, Models, and Monitors. Environ. Sci. Technol. 2019, 53, 2595-2611. [CrossRef]

28. Van Donkelaar, A.; Martin, R.V.; Brauer, M.; Hsu, N.C.; Kahn, R.A.; Levy, R.C.; Lyapustin, A.; Sayer, A.M.; Winker, D.M. Global Estimates of Fine Particulate Matter using a Combined Geophysical-Statistical Method with Information from Satellites, Models, and Monitors. Environ. Sci. Technol. 2016, 50, 3762-3772. [CrossRef]

29. Liu, Y.; Wu, J.; Yu, D.; Ma, Q. The relationship between urban form and air pollution depends on seasonality and city size. Environ. Sci. Pollut. Res. 2018, 25, 15554-15567. [CrossRef]

30. Zhou, J.; Xing, Z.; Deng, J.; Du, K. Characterizing and sourcing ambient PM2.5 over key emission regions in China I: Water-soluble ions and carbonaceous fractions. Atmos. Environ. 2016, 135, 20-30. [CrossRef]

31. Li, G.; Fang, C.; Wang, S.; Sun, S. The Effect of Economic Growth, Urbanization, and Industrialization on Fine Particulate Matter (PM2.5) Concentrations in China. Environ. Sci. Technol. 2016, 50, 11452-11459. [CrossRef] [PubMed]

32. Łowicki, D. Landscape pattern as an indicator of urban air pollution of particulate matter in Poland. Ecol. Indic. 2019, 97, 17-24. [CrossRef]

33. Zhang, R.; Wang, G.; Guo, S.; Zamora, M.L.; Ying, Q.; Lin, Y.; Wang, W.; Hu, M.; Wang, Y. Formation of Urban Fine Particulate. Matter. Chem. Rev. 2015, 115, 3803-3855. [CrossRef] [PubMed]

34. Grossman, G.M.; Krueger, A.B. Economic Growth and the Environment. Quat. J. Econ. 1995, 110, $353-377$. [CrossRef]

35. Shen, Y.; Lisa Ahlers, A. Local environmental governance innovation in China: Staging 'triangular dialogues' for industrial air pollution control. J. Chin. Gov. 2018, 3, 351-369. [CrossRef]

36. Li, F.; Zhou, T. Effects of urban form on air quality in China: An analysis based on the spatial autoregressive model. Cities 2019, 89, 130-140. [CrossRef]

37. Xu, L.; Batterman, S.; Chen, F.; Li, J.; Zhong, X.; Feng, Y.; Rao, Q.; Chen, F. Spatiotemporal characteristics of $\mathrm{PM}_{2.5}$ and $\mathrm{PM}_{10}$ at urban and corresponding background sites in 23 cities in China. Sci. Total Environ. 2017, 599-600, 2074-2084. [CrossRef]

38. Han, L.; Zhou, W.; Li, W.; Li, L. Impact of urbanization level on urban air quality: A case of fine particles $\left(\mathrm{PM}_{2.5}\right)$ in Chinese cities. Environ. Pollut. 2014, 194, 163-170. [CrossRef]

39. Liu, C.; Henderson, B.H.; Wang, D.; Yang, X.; Peng, Z. A land use regression application into assessing spatial variation of intra-urban fine particulate matter $\left(\mathrm{PM}_{2.5}\right)$ and nitrogen dioxide $\left(\mathrm{NO}_{2}\right)$ concentrations in City of Shanghai, China. Sci. Total Environ. 2016, 565, 607-615. [CrossRef]

40. Zhao, X.; Zhang, X.; Xu, X.; Xu, J.; Meng, W.; Pu, W. Seasonal and diurnal variations of ambient $\mathrm{PM}_{2.5}$ concentration in urban and rural environments in Beijing. Atmos. Environ. 2009, 43, 2893-2900. [CrossRef] 
41. Nowak, D.J.; Hirabayashi, S.; Bodine, A.; Greenfield, E. Tree and forest effects on air quality and human health in the United States. Environ. Pollut. 2014, 193, 119-129. [CrossRef] [PubMed]

42. Lin, Y.; Yuan, X.; Zhai, T.; Wang, J. Effects of land-use patterns on $\mathrm{PM}_{2.5}$ in China's developed coastal region: Exploration and solutions. Sci. Total Environ. 2020, 703, 135602. [CrossRef] [PubMed]

(C) 2020 by the authors. Licensee MDPI, Basel, Switzerland. This article is an open access article distributed under the terms and conditions of the Creative Commons Attribution (CC BY) license (http://creativecommons.org/licenses/by/4.0/). 